\title{
Fundamentality, Effectiveness and Objectivity of Gauge Symmetries
}

\begin{abstract}
Aldo Filomeno*
Abstract

Much recent philosophy of physics has investigated the process of symmetry breaking. Here, I critically assess the alleged symmetry restoration at the fundamental scale. I draw attention to the contingency that gauge symmetries exhibit, i.e. the fact that they have been chosen from among a countably infinite space of possibilities. I appeal to this feature of group theory to argue that any metaphysical account of fundamental laws that expects symmetry restoration up to the fundamental level is not fully satisfactory. This is a symmetry argument in line with Curie's $1^{\text {st }}$ principle. Further, I argue that this same feature of group theory helps to explain the "unreasonable" effectiveness of (this subfield of) mathematics in (this subfield of) physics, and that it reduces the philosophical significance that has been attributed to the objectivity of gauge symmetries.
\end{abstract}

\section{Keywords}

Metaphysics of Fundamental Laws, Gauge Symmetry, Symmetry Restoration, Symmetry Arguments

*Aldo Filomeno is at the Instituto de Investigaciones Filosóficas, Universidad Nacional Autónoma de México. Correspondence to: Instituto de Investigaciones Filosóficas, Universidad Nacional Autónoma de México, Circuito Mario de la Cueva s/n, Ciudad Universitaria, C.P. 04510, Coyoacán, CDMX, Mexico. E-mail: aldofilomeno@filosoficas.unam.mx 


\section{Highlights:}

- Intrinsic features of group theory suggest that a metaphysics of fundamental laws consisting of bigger local gauge symmetries welcomes further explanation.

- Said features help to explain the astonishing effectiveness of group theory in particle physics.

- Said features lessen any philosophical significance of the objectivity associated with gauge symmetry.

\section{Contents}

1 Introduction $\quad 3$

2 Preliminary Assumptions 6

3 The "Unreasonable" Success of (Gauge) Symmetry Principles in Physics 8

4 The Contingency of Gauge Invariance - the Case of the Strong Interaction

5 Significance of the Contingency of Gauge Invariance 16

5.1 The Reasonable Effectiveness of Group Theory in Particle Physics 16

5.2 The Significance of the Objectivity . . . . . . . . . . . 18

5.3 The Fundamental Metaphysics that stems from Symmetry Restoration ...................... 20

6 Conclusion $\quad 23$

Acknowledgements

For their helpful comments I wish to thank Elías Okon, Carl Hoefer, Keizo Matsubara, Kerry McKenzie, María Martinez del Rosario, Auro Michele, David Rey, Carlos Romero, Giulio Tirabassi, and an anonymous referee. I am grateful to audiences at the 'Seminario de investigadores' at UNAM and at the 'CauSci final conference' in Oslo. This research has been economically supported by the 'Instituto de Investigaciones Filosóficas' of the Universidad Autónoma de México through a fellowship from the postdoctoral fellowship program DGAPA-UNAM. 


\section{Introduction}

As recent philosophical investigations have shown, distinctive features of YangMills gauge theories can be significant for the philosophical interpretation of the laws they describe. ${ }^{1}$ In this paper, we will explore the philosophical significance of one of these features. The resulting assessment is intended to attenuate the enthusiasm that surrounds the predominant role of gauge symmetries in particle physics. After the privileged status that was conferred to global continuous symmetries - deemed by Wigner (1967) as meta-laws or "super-principles"-the astonishing success of local gauge symmetries in the constitution of modern particle physics bolstered the enthusiasm. I sketch why this has been so in section 3 . The aim of this paper is, in general terms, to attenuate this enthusiasm. The aim, thus, is to target a widespread attitude: that of those who, satisfied by the elegance and beauty (and by other virtues outlined in section 3) of the symmetries that describe the dynamics of the world, seek no further explanation for such laws. I argue that this attitude should change, as some have previously argued, albeit from substantially different perspectives (e.g. Peirce (1867), Weinberg (1981), Wheeler (1982), Froggatt and Nielsen (1991), or Unger and Smolin (2014)). ${ }^{2}$

More specifically, the aim is to attenuate the widely held satisfaction with the postulation - well motivated by a variety of scientific and philosophical reasonsof bigger and bigger symmetry groups in higher energy regimes. No gain will be achieved, I argue, in our comprehension of certain metaphysical issues about laws of nature in case we arrive to a Theory of Everything (ToE) whose fundamental interactions are described by local gauge symmetries. ${ }^{3}$

This first conclusion targets physicists who seem to believe that the final laws will be self-explanatory and will naturally dilute the current philosophical

\footnotetext{
${ }^{1}$ See e.g. Kantorovich (2003, 2009), Baker (2010), McKenzie (2012, 2013), Nounou (2015), or Filomeno (2014, ch. 1, 2).

${ }^{2}$ I briefly compare their approaches with mine at the end of the paper.

${ }^{3}$ I thus refer to any present or future theory based on what nowadays is our best empirically tested physical theory, Quantum Field Theory (QFT). This includes ToE's such as Superstring Theory in its many variants, E8's proposal, and ToE's based on Canonical and Loop Quantum Gravity. More details in section 2.
} 
questions regarding the nature of laws. Yet it also targets (certain) naturalistic metaphysicians as well as primitivists about laws. Naturalistic metaphysicians rely on our best physics to inform us about metaphysical issues-such as, say, our comprehension of the nature of laws. They are well aware that of course future physics may change our metaphysical picture of the world, but the best we can do at the present day is to rely on what our current physics is telling us. However, this first conclusion warns us about principled limitations of the naturalistic approach, in that even future ToE's will leave unsettled certain philosophical issues about the nature of laws. ${ }^{4}$ Similarly, primitivists about laws are targeted, insofar as I argue that these philosophical issues should be explained. So, while interestingly some have argued that certain characteristics of current physical laws help to explain certain philosophical aspects of laws (see footnote 1), I argue that certain characteristics of current physical laws suggest that some philosophical questions will not be answered.

The way I argue for this conclusion is the following. In section 4, I examine in detail a characteristic of the local gauge symmetries ubiquitous in particle physics. In short, this feature refers to diverse dimensions of contingency of the mathematical representation: the fact that they have been chosen from among different layers of infinite possibilities. I spell this feature out by means of the specific case study of the Lie group SU(3) and the strong nuclear interaction. Then, in section $5 \mathrm{I}$ defend three philosophical consequences stemming from such a feature: the first conclusion advanced above and two others. In what remains of this section I explain more in detail the three conclusions of this paper.

First, I argue that such feature of group theory diminishes the philosophical significance sometimes conferred to the objectivity of the laws written in terms of Lie groups - the objectivity implicit in the notion of symmetry was originally highlighted by Weyl (1952), and has been recently stressed in the literature on ontic structural realism, where the appeal to group theory is frequent (Ladyman, 2014, sec. 4.1); see also Nozick (2001), Earman (2003), Kosso (2003), Debs and

\footnotetext{
${ }^{4}$ Critical assessments of naturalistic metaphysics are a hot topic today. See e.g. (French and McKenzie, 2015), (Ross et al., 2015), or (Tahko and Morganti, 2016).
} 
Redhead (2009).

Second, I argue that said feature makes the astonishing success of group theory in particle physics not unreasonable but, on the contrary, to be expected. Thus, this second argument adds up to a certain literature in philosophy of mathematics that aims to explain the unreasonable effectiveness of mathematics in physics; e.g. Bueno (2011), Räz and Sauer (2015), or Pincock (2004). This literature explores the idea of a mapping of the structure of the world with the structures described by mathematics; I support this idea by spelling out how this mapping obtains in the especially astonishing case of the effectiveness of gauge symmetries in particle physics.

Finally, again from said characterization of section 4 I defend the conclusion advanced at the beginning: I argue that the bigger symmetry groups conjectured by current ToE's, when intended to play the role of fundamental laws, welcome further philosophical elucidation. Let me specify in what sense. This last conclusion relies on a premise that states that it is asymmetry, not symmetry, that is to be associated with the notion of design and hence demands explanation. This premise is elaborated and defended by Kosso (2003), and is in line with Curie's first principle. My argument, then, is the following. Given that the characterization of group theory of section 4 shows that bigger symmetry groups will not correspond to a state of absolute dynamical symmetry but will still display a certain degree of asymmetry, the symmetry argument of Kosso (2003) will lead us to conclude that bigger symmetry groups, in the role of fundamental laws, welcome further explanation.

Before, in the next section 2 I begin by making some assumptions. Notably, these assumptions help my opponent-i.e., if any of them were false, it would bolster the conclusions of the paper. So, while they are plausible assumptions, I also cite how some of them have been disputed. Then in the next sections we will leave them aside, and we will focus on the main arguments aforementioned. 


\section{Preliminary Assumptions}

In what follows, I will assume the necessary condition for fundamentality in QFT that the theory is asymptotically safe. Asymptotic safety is the condition that in the ultraviolet regime all the coupling constants must possess a finite value; that is, the QFT has to be well defined at all energies without being perturbatively non-renormalizable. In other words, a QFT must correspond to a trajectory of the renormalization group that converges asymptotically to a fixed point in the UV regime. This technical requirement is necessary to be able to speak of a fundamental ToE rather than of a tower of autonomous effective field theories (for philosophical debates about effective field theories, see e.g. Castellani (2002), Cat (1998), or Cao (1988)).

Second, I will assume that the prospect of a future unification of all the elementary interactions succeeds. The failure of unification would aggravate the dissatisfaction with the resulting metaphysical picture, in line with what I will argue. And let me note that a successful unification faces several challenges: Maudlin (1996) and Morrison (2000, 2013) highlight diverse threats. For instance, Morrison $(2013,383)$ (and similarly Maudlin $(1996,143)$ ) points out that the Standard Model is not a truly unified theory with a single group but an amalgam of three groups $U(1) \otimes S U(2) \otimes S U(3)$, and that unification presupposes that gravity corresponds to an elementary interaction like the others, whereas General Relativity successfully explains gravity without the postulation of such a force-carrier (the effects of gravitation are due to spacetime curvature).

Third, I will assume that the unnaturalness of QFT is resolved in future ToE's. This is because the characterization of gauge symmetries that I give in section 4 highlights their non-trivial degree of ad hoc contingency. Thus, the lack of naturalness of QFT can be seen to support this characterization. Roughly, the notion of unnaturalness is related with the apparently ad hoc (and fine-tuned) values of many parameters. The idea behind is that of putting in by hand the parameters in order to enable the model to obtain empirical adequacy. An overarching definition is found in (Williams, 2015), where naturalness is defined as the lack of sensitive 
correlations between widely separated physical scales. ${ }^{5}$ The already existent unnaturalness increased after the experiments carried out at the Large Hadron Collider, where the detection of the Higgs boson in the scale of $125 \mathrm{GeV}$ is ruling out the most natural versions of Supersymmetry, such as the Minimal Supersymmetric Standard Model.

Fourth, I will adopt an ontological interpretation of local gauge symmetries. This means that, paraphrasing Wigner, local gauge symmetries have an ontological "active" role and provide physically significant claims about the carvings of nature. The falsity this assumption amounts to an interpretation of gauge symmetries as redundant surplus mathematical structure (as in Wigner (1967), Ismael and Van Fraassen (2003) or Redhead (2003)). If this assumption is false, two of my conclusions are more easily met: if symmetries are not physically significant, there will be a further reason to demand that ToE's welcome further philosophical elucidation, and the objectivity of symmetries will be of less philosophical significance.

Last but not least, I will consider that the process of (both explicit and spontaneous) symmetry-breaking is well understood. There is a variety of philosophical literature discussing the process of symmetry breaking; e.g. Kosso (2000), Morrison (1995, 2003), Castellani (2003), Earman (2003), Strocchi (2012), or Friederich (2013). For instance, I will assume that the spontaneous breaking of a symmetry does make sense without needing to presuppose an unknown hidden law that is pushing the system to execute the phase transition from one group to one of its subgroups, or that those hidden unobserved postulated bigger symmetries really correspond to more fundamental true laws. Flaws of this sort would bolster my critical assessment of a metaphysical picture of fundamental laws based on the restoration of gauge symmetries in higher energy scales.

The next section 3 briefly recaps some of the novel and non-trivial virtues of the gauge paradigm that have led to the enthusiasm mentioned in section 1, which

\footnotetext{
${ }^{5}$ In addition to Williams (2015), for a philosophical assessment of the notion of naturalness cf. Friederich et al. (2014). Other discussions around unnaturalness include Borrelli (2011), Feng (2013), Evans et al. (2014), and Fowlie (2014).
} 
I critically assess in the rest of the paper.

\section{The "Unreasonable" Success of (Gauge) Symme- try Principles in Physics}

As contended decades ago by Wigner (1967), global continuous symmetry principles could be plausibly considered some sort of "superprinciples", in the sense of some kind of necessary or a priori meta-laws. The advent of more symmetries of a new type, local (also called 'internal') and following the so called gauge principle, has bolstered the enthusiasm and has been taken as a sign of the "elegance of nature" (Wilczek, 2008, 63). ${ }^{6}$ Remarkably, it turns out that nowadays all the elementary interactions of the Standard Model can be described according to this procedure.

Furthermore, physicists have formulated the theories as a result of conceptual (mathematical) work much before the posterior solid experimental support: see Bangu $(2013,2008)$ for the study of impressive historical cases, like the prediction of the $\Omega^{-}$boson. Moreover, it turns out that the gauge paradigm exhibits an appealing simplicity in that few inputs are required to specify full theories (Martin, 2003, p.53). This, in turn, leads us to one of the most attractive features of this new physics: its unificatory role. All elementary interactions (though gravitation only in theory) are described in terms of local gauge symmetries.

Additionally, as I will discuss in section 5, the distinctive kind of invariance that group theory is able to express has been taken to characterize the notion of objectivity (Ladyman, 2014, sec. 4.1). This characterization allegedly allows physics to get rid of the confines of a particular coordinate system.

Martin $(2003,41)$ describes the enthusiastic attitude:

\footnotetext{
${ }^{6}$ The gauge principle specifies a procedure for obtaining an interaction term in the Lagrangian which is symmetric with respect to a continuous symmetry. The results of localizing (or 'gauging') the global symmetry group involves the introduction of additional fields so that the Lagrangian is extended to a new one that is covariant with respect to the group of local transformations.
} 
'the 'gauge philosophy' is often elevated and local gauge symmetry principles enshrined. Gauge symmetry principles are regularly invoked in the context of justification, as deep physical principles, fundamental starting points in thinking about why physical theories are the way they are, so to speak. This finds expression, for example, in the prominent current view of symmetry as undergirding our physical worldview in some strong sense"

and (ibidem, 52):

"gauge invariance is often invoked as a supremely powerful, beautiful, deeply physical, even undeniably necessary feature of current fundamental physical theory"

In sum, all these features have been central reasons for the enthusiasm towards this new physics and the judgements of (both vaguely stated) elegance and necessity. However, having outlined some key points of the particular success and novel features of gauge symmetry principles, let's examine in depth another feature of group theory to show that this success should not be interpreted in too strong a manner.

\section{The Contingency of Gauge Invariance - the Case of the Strong Interaction}

In this section, I explain in detail the multiple layers of contingency of local gauge symmetries. The three arguments that I propose in section 5 appeal to this feature. I make it explicit by means of a specific case, that of the strong interaction. This feature makes evident that the particular type of symmetry groups constituting the Standard Model of particle physics are, in spite of their remarkable virtues, not a priori reasonable nor necessary at all, but significantly contingent and chosen due to empirical adequacy among a large space of possibilities. In addition to this well known feature, we will see that sometimes something stronger holds: group-theoretic underdetermination, that is, empirical adequacy and mathematical constraints do not uniquely determine the specific local gauge group. 
First of all, though, bear in mind that my focus is on gauge symmetry, not on other kinds of symmetry principles. In fact, a strong metaphysical interpretation might be given of the symmetry principles that Wigner dubbed 'geometrical' (unlike those dubbed 'dynamical', to which gauge symmetries belong). The 'geometrical' concern the invariance of all the laws of nature under geometric transformations tied to regularities of the underlying spacetime, while the 'dynamical' concern the invariance of the form (also labeled as covariance) of the laws governing particular interactions under groups of transformations not tied to spacetime (Martin, 2003, 50). It is the former type which was contended by Wigner (1967) to be akin to meta-laws, "almost necessary prerequisites" for laws.

With this in place, let's focus now on one of the elementary interactions, the strong nuclear interaction, which displays one of the local gauge symmetries of the Standard Model, the color local gauge invariance of quarks. The color invariance is represented by the symmetry group $\mathrm{SU}(3)$, the Special Unitary group of degree 3. The theory of Quantum Chromo Dynamics (QCD) successfully describes the color strong interaction: the property named 'color' is conserved due to a certain type of bosons (force carrier particles) called gluons, exchanged in the interactions. Each of them carries one unit of color and one unit of anticolor; thus they guarantee the conservation of the initial color that changes in the quark in a 'strong' interaction with another quark. The invariance is achieved by adding a new term in the lagrangian that corresponds to a gauge field, so that the lagrangian becomes invariant under the operations of the group. ${ }^{7}$ There are 3 charges of this color interaction, named: 'red', 'green', and 'blue'. Therefore, there are nine logically possible combinations of the 3 colors: $r \bar{r}, r \bar{g}, r \bar{b}, b \bar{r}, b \bar{g}, b \bar{b}, g \bar{r}, g \bar{b}, g \bar{g}$.

Then, every symmetry group has the so called 'representations', and it is always the so called 'adjoint representation' that describes the force carriers, in this case the gluons. ${ }^{8}$ The adjoint representation of $\mathrm{SU}(3)$ is not nine but eight

\footnotetext{
${ }^{7}$ For a detailed presentation of the mathematical machinery behind see Griffiths (2008, ch. 8), Robinson et al. (2008, Part II - Algebraic foundations (esp. 2.2.15)), and Cottingham and Greenwood (2007, ch. 16).

${ }^{8}$ A representation of a Lie group is one of the ways of representing the elements of the group as linear transformations of the group's Lie algebra, where the elements constitute a vector space.
} 
dimensional. In this representation the nine states are structured in an octet and the other state is a singlet element apart. The linearly independent base vectors that constitute the octet can be written as ${ }^{9}$ :
$|1\rangle=(r \bar{b}+b \bar{r}) / \sqrt{2}$
$|5\rangle=-i(r \bar{g}-g \bar{r}) / \sqrt{2}$
$|2\rangle=-i(r \bar{b}-b \bar{r}) / \sqrt{2}$
$|6\rangle=(b \bar{g}+g \bar{b}) / \sqrt{2}$
$|3\rangle=(r \bar{r}-b \bar{b}) / \sqrt{2}$
$|7\rangle=-i(b \bar{g}-g \bar{b}) / \sqrt{2}$
$|4\rangle=(r \bar{g}+g \bar{r}) / \sqrt{2}$
$|8\rangle=(r \bar{r}+b \bar{b}-2 g \bar{g}) / \sqrt{6}$

and the singlet element is:

$|9\rangle=(r \bar{r}+b \bar{b}+g \bar{g}) / \sqrt{3}$ The combination $r \bar{r}+b \bar{b}+g \bar{g}$ is not verified in exper-

Figure 1: The pattern of strong charges for the three colors of quark, three antiquarks, and eight gluons (in black) with two of zero charge overlapping in the center. The vertical axis is strangeness and the horizontal is isospin.

iment (Griffiths, 2008, 285); later I will come back to this detail. Thus, the eight gluons that exist in nature are described by the eight so called 'generators' that

Then, an adjoint representation is defined as a (finite-dimensional irreducible) representation in which the structure constants themselves form a representation of the group. (The 'structure constants' of a Lie group determine the commutation relations between its generators in the associated Lie algebra).

9 The states are added in linear combinations according to the principle of superposition of Quantum Mechanics. The numerical parameters are required for normalization. 
compose the octet, the set of linearly independent vectors above that form a vector base of the group SU(3). Each generator aims to represent the color state of a certain type of gluon. ${ }^{10}$ The situation is beautifully illustrated in figure 1 . The octet of the figure illustrates indeed the existence of a tight pattern between the gluons (and with the quarks).

Now, to what extent should we "celebrate" the beautiful and unified pattern exhibited between the gluons? In the next paragraphs I argue that we should not celebrate too much, as the mathematical model is not mysteriously successful nor necessary nor a priori reasonable as these aesthetic patterns and all that has been said in section 3 might suggest.

The first thing to note is that different gauge fields and different couplings between the gauge fields and matter fields lead to different local symmetry groups. Thus, we can have a classification of all the possible gauge fields which can exist in a universe in function of the possibility space of such groups.

Then, the most general constraints upon a free (i.e. non interacting) system are the following. In QFT a free particle can be any irreducible, projective, unitary representation of the local space-time symmetry group (see McCabe $(2011,6)$ for the details). This group is the largest possible local symmetry group of our actual Minkowski space-time, and is the the semi-direct product $S L(2, \mathbb{C}) \ltimes \mathbb{R}^{3,1}$ (McCabe, 2011, 16). ${ }^{11}$ The constraint of this group determines a whole set of possible families of free particles that could exist in the world. It is the specific actual values of masses and spins of the actual universe that single out the actual

\footnotetext{
${ }^{10}$ In general, the force-carriers correspond to the eigenvectors of the generators, while the eigenvalues of these eigenvectors are the physically measurable charges (color, in our case).

${ }^{11}$ This group is determined by the spatiotemporal dimension, the geometric signature, and the spatial and temporal local orientation of our world. Such group is determined by the large-scale structure of a space-time, which is described by a pseudo-Riemannian manifold $(\mathrm{M}, g)$, in our actual world of dimension 4 (Lorentzian manifold, isomorphic to Minkowski space-time), and a metric of 3 spatial +1 temporal dimensions. More exactly, the group is the so called restricted Poincaré Group and is $O(3,1) \ltimes \mathbb{R}^{3,1}$ (McCabe, 2011, 16). The simply connected version of $O(3,1) \ltimes \mathbb{R}^{3,1}$ is the so called universal covering group, which is $S L(2, \mathbb{C}) \ltimes \mathbb{R}^{3,1}$. I am simplifying not introducing the further restrictions of local space orientation and local time orientation. To the interested reader I refer to McCabe $(2011,38)$.
} 
free elementary particle types.

Then, further restrictions on the free particles come from the gauge symmetries of the elementary interactions, like the mentioned SU(3). The groups constituting the elementary interactions of the Standard Model must belong to the class of compact, simple, simply connected Lie groups; so, what is the possibility space of this class? This has been classified in the 'Cartan classification' (Lederman and Hill, 2004, 315); cf. (Simon, 1995, 151 Table VII.1):

1. Rotational symmetries of spheres that live in $\mathrm{N}$ real coordinate dimensions: $\mathrm{O}(2)=\mathrm{U}(1), \mathrm{SO}(3)=\mathrm{SU}(2), \mathrm{SO}(4), \mathrm{SO}(5), \ldots, \mathrm{SO}(\mathrm{N}), \ldots$

2. Rotational symmetries of spheres that live in $\mathrm{N}$ complex coordinate dimensions: $\mathrm{U}(1), \mathrm{SU}(2), \mathrm{SU}(3), \mathrm{SU}(4), \ldots, \mathrm{SU}(\mathrm{N}), \ldots$

3. Symplectic groups, which are the symmetries of $\mathrm{N}$ harmonic oscillators: $\operatorname{Sp}(2), \operatorname{Sp}(4), \ldots, \operatorname{Sp}(2 \mathrm{~N}), \ldots$

4. The 'exceptional' groups: $G_{2}, F_{4}, E_{6}, E_{7}$, and $E_{8}$

As it appears, the resulting landscape is infinite; there is a countably infinite number of possible Lie groups available. Thus, this classification allows us to realize the first dimension of the contingency of the symmetry groups chosen: in the description of an elementary interaction, $S U(3)$ is just one of the infinite possible symmetry groups at our disposition.

This first layer of contingency is further bolstered by past episodes in the history of particle physics, before the solid empirical confirmation of the current theory: episodes involving the possibility of a ninth gluon. We have seen that the singlet element above corresponds to one of the nine logical possibilities, but that it does not appear to exist. One can then wonder why there are eight gluons instead of nine (in fact, see e.g. Griffiths (2008, 285) or Bottomley and Baez (1996)). Indeed, such a ninth gluon could have existed; a nine-gluon theory is perfectly possible in principle, but it would describe a world very different from the actual (Griffiths, 2008, 285). In that case, it would be as common and conspicuous as the photon, and Griffiths $(2008,303)$ considers the case that the ninth gluon would 
have corresponded to the photon. This is now discarded but it was investigated in the eighties (Fischbach et al., 1986). ${ }^{12}$ The adjoint representation of SU(3), in this scenario, could have been properly used to describe the situation; the ninth gluon would have corresponded to the singlet state of SU(3). Thus, the group SU(3) is not univocally correspondent with the actual world but is at least compatible with two different possible worlds. ${ }^{13}$

Likewise, in the possible world of nine gluons the symmetry group of QCD could have been other than SU(3). Specifically, a theory of nine gluons can be also described by the group U(3) (Griffiths, 2008, 286). The experimental results that discover eight gluons require us to discard an alternative such as U(3). This is a usual way scientific practice is carried out, and so it has to be ( $\mathrm{I}$ am of course not disputing this procedure of scientific research). To remark this practice in this field of physics is aimed to show that there is no a priori reason-no sufficient mathematical constraints - to prefer the group SU(3) over U(3). In sum, we have identified, in addition to the first layer of contingency-i.e., the contingency in the choice of a specific group from a countable infinity of groups - a situation of group-theoretic underdetermination.

A further layer of contingency becomes evident when we focus not on the gauge force fields (the bosons, such as the gluons described by the SU(3) group), but on the gauge matter fields (the fermions, such as the quarks, also described by the $\mathrm{SU}(3)$ group) and on the possibility space of representations. While for the bosons the representation chosen is always unique, namely the adjoint represen-

\footnotetext{
12 In (Griffiths, 2008, 303, Problem 8.11) it is explained how the situation would look like: the gluon would couple to all baryons with the same strength, not, as the photon does, in proportion to their charge. In the end this would look like as an extra contribution to gravity, contrary to actual evidence.

${ }^{13}$ The phenomenon of confinement states that all naturally occurring free particles have to be color singlets. Correspondingly, the gluons of the octet are not free particles. Instead, $|9\rangle$ is a color singlet, and if it would exist as a mediator it should be a free particle. We do not know a priori that this is not the case, so it is empirical evidence that makes us discard this option. Indeed, if $|9\rangle$ would exist it could be exchanged between two color singlets-a proton and a neutron, say_bringing about a long range coupling of the color strength (Griffiths, 2008, 286), but this is contrary to actual evidence.
} 
tation, for the fermions the physically significant representations are the so called 'irreducible representations'. The states in the irreducible representation are those that possess the determinate properties measured in reality, like isospin and hypercharge for the case of SU(3). Informally, the irreducible representations of a group are the representations of the smallest possible order, i.e., those that cannot be further reduced (more technically, they are said to have no nontrivial invariant subspaces). The connection of a symmetry group with physical reality is made through the choice of an irreducible representation of the group. Thus, there is a mapping of the irreducible representation with a physical interpretation of families of matter particles (fermions) that could exist in the world. It turns out that there are countably infinite representations of this type, for SU(3) as well as for any other group. Therefore, there are infinite possible classes of sets of particles (the particle multiplets) allowed for each of the (in turn infinite) symmetry groups. The moral I want to draw is that, in the end, the particular final choice is made from among an extremely vast space of possibilities.

In conclusion, I want to underline that, pace the virtues of the new physics portrayed in section 3 , the mathematical description of the strong interaction is a contingent representation chosen from among a wide space of possibilities, and is not a priori reasonable nor (logically or metaphysically) necessary in spite of the mathematical constraints, of its elegance, of the unification with the rest of interactions, and of the theoretical predictions much before the astonishing empirical success. Along with the particular case of SU(3), of course a similar diagnosis can be extended to the rest of gauge invariances, from those that describe the elementary interactions of the Standard Model, the product $U(1) \otimes S U(2) \otimes S U(3)$, to, say, the exceptional group $E_{8} \cdot{ }^{14}$

Martin $(2003,52)$ shares a similar diagnosis "against" gauge invariance. He remarks other factors that have to be taken into account when a gauge-invariant

\footnotetext{
${ }^{14}$ The stronger claim of underdetermination, illustrated before with the cases of SU(3) vs. U(3), might not generalize to other groups. We need not: the coming arguments hinge on the weaker feature that I have dubbed as the contingency of gauge symmetry; i.e., the contingency in the choice of a specific group from different layers of infinite possibilities. Thanks to an anonymous referee for raising this point.
} 
term is added into the lagrangian: Lorentz invariance, simplicity, and renormalizability. His main upshot is to highlight the heuristic character of such symmetries, showing how "the gauge fields are put in by hand to large extent" (ibidem, 45). See also Bangu (2013, 298), who wonders about the metaphysical and epistemological grounds upon which to demand local gauge invariance or, as he puts it, to demand the justification of the Yang-Mills gauge principle.

\section{Significance of the Contingency of Gauge Invari- ance}

After the previous characterization of gauge symmetries we can realize the lack of any a priori reasonableness or necessity stronger than physical necessity. In spite of the virtues previously portrayed, such a strong interpretation is deferred to Wigner's geometrical invariances, if at all. With this in place, from the characterization of the previous section we can now pursue the conclusions mentioned at the beginning of the article.

\subsection{The Reasonable Effectiveness of Group Theory in Particle Physics}

The first conclusion regards the intimate relationship between group theory and the physical world, a conclusion that adds up to the the more general debate about the mysterious, or "unreasonable" (Wigner, 1960), effectiveness of mathematics in physics. Among the several dimensions that can raise our astonishment, I focus here only on the astonishing empirical success (heuristically, at least) of group theory in describing the world of particle physics (for other puzzles around the effectiveness of mathematics see Wigner (1960)). Assuming an ontological interpretation of gauge symmetries that grants them physical significance, the central question is: Why is group theory so central to describing part of the physical 
world ${ }^{15}$

Our characterization of the previous section can help to illustrate the accounts that explain the effectiveness of mathematics in physics in virtue of a mapping of the structure of the world with the structures described by mathematics-as e.g. in (Bueno, 2011), (Räz and Sauer, 2015) or (Pincock, 2004). The underlying idea of this type of accounts is roughly the following.

First, we have to distinguish two different issues that should not be conflated. One is the fact that the physical world displays stable spatiotemporal patterns; that is, the world is ordered, displaying stable patterns of behaviour - what the laws of physics aim to describe. We have to assume this uncontroversial (yet philosophically compelling) fact, and distinguish it from the likewise puzzling fact that mathematics seems unreasonably effective to describe the world. Now, for the sake of exposition, consider a structuralist approach to mathematics, whether platonist or nominalist (e.g. Shapiro (1997) or Hellman (1989) respectively)—for present purposes, it does not matter which. As the previous section 4 has emphasized, mathematics is a language full of non-actualized structures; in particular, we have seen that group theory describes a wide range of actualized as well as non-actualized structures. While the vast majority of these structures will not correspond to the actual structures/patterns of the world, within this extremely wide space of possibilities it is not unreasonable to expect that a subset of these abstract patterns matches some of the actual patterns of nature. So, the specific groups that actually constitute our best physical theories are only some of the infinite possible mathematical descriptions of the regularities of a world. Understood this way, we can see how it is not unreasonable to expect that part of the world investigated by the physical sciences is going to be described by group theory. ${ }^{16}$

\footnotetext{
15 The question could be generalized to the whole of mathematics, asking not only about group theory but also about Hilbert spaces, riemannian geometry, differential calculus, and so on and so forth. Here, though, the focus is only on group theory — an especially interesting subfield of mathematics due to its predominant role in particle physics and its apparent virtues and privileged status (see sec. 3).

16 The huge expressive power of group theory is what makes its effectiveness in physics more reasonable. It is not the other way around: it is not that given the abundance of possibilities is
} 
This line of thought, supported by the characterization of section 4, does not need to confer any privileged ontological status neither to mathematical entities nor to the laws of physics. As such, it can be considered a better (or at least more economical) explanation than that of those that, too much nurtured by the effectiveness of mathematics (and the other virtues portrayed in section 3), postulate more abundant ontologies. Extreme examples of the latter are the several variants that confer existence to mathematics in the world, such as Tegmark (2014). Also, ontic structural realists want to be realists about the gauge symmetries in their well-known strong sense: the structures represented by the actual Lie groups of particle physics constitute the fundamental furniture of the world. The explanation now sketched, though, aims to dissolve any privileged ontological status ascribed to the Lie groups in case the justification for such a status comes from their astonishing effectiveness in particle physics.

Still, ontic structural realists invoke group theory (there is even the variant of 'Group Structural Realism' (Roberts, 2008)) on other grounds; for example, by emphasizing that the structures that group theory represent bear a special relation with the cherished notion of objectivity, a notion that we certainly want to confer to our best science and to our best metaphysical theories. This brings us to the second main remark of this paper.

\subsection{The Significance of the Objectivity}

The characterization of gauge symmetries of the previous section bears on the objectivity that has been traditionally associated with the notion of symmetry. The objectivity of a fact, in general, is taken to guarantee its independence from different perspectives, agent's beliefs, desires, observations, or measurements, as well as to guarantee a lack of rational intersubjective disagreement about it (French $(2014,161)$ who draws from Earman (2003), in turn from Nozick (2001); see also even more unreasonable that one of them applies (this line of thought would mirror that of the lottery paradox). It is just because we have at our disposition a rich language (group theory) that is reasonable to expect that one of its many possible expressions (groups) will be capable of describing a certain pattern of nature. 
the notion of 'true generality' of Van Fraassen (1989, ch. 11 esp. sec. 5)). Then, Weyl (1952) famously defined objectivity as invariance with respect to the relevant group of automorphisms. In particular, that the invariance that group theory expresses can be taken to characterize the notion of objectivity has been stressed (again) by advocates of ontic structural realism (Ladyman (2014, 4.1), Ladyman and Ross (2009, 3.3), French (2014, 6.3), Lyre (2004)). Within the metaphysical framework of OSR, the question we are now going to address is: Does said objectivity have any philosophical significance such that it provides any distinctive support to OSR? Or more generally, not restricted to this particular metaphysical view: To what extent does the sort of objectivity indeed exhibited by the gauge symmetries is of any substantial significance for a metaphysical account of fundamental laws so constituted? Or, in other terms: Should we be more satisfied with the resulting laws given their alleged objectivity?

Admittedly, the notion of objectivity has been applied to the invariance of relations among properties, not to properties or objects, so this fact would help the ontic structural realist to dispense with any commitment to fundamental objects (along this line see Rickles and French (2003)). However, the characterization of gauge symmetries of the previous section diminishes any substantive philosophical significance of the objectivity that is (in fact) attained by the elementary interactions described in terms of Lie groups; for the kind of contingency highlighted in the previous section leads us to admit that, regardless of the degree of objectivity attained by a particular group (like our SU(3)), there are still infinite other possible equally objective groups.

This argument is similar to what Debs and Redhead (2009) stress when they remark that one should be able to sort out which symmetry is physically significant amongst all the possible symmetries - at least, which is heuristically fruitful. ${ }^{17}$ In response to this demand, French $(2014,161)$ (see also McKenzie $(2014,375$ 6), French and Ladyman (2003, 75), and French (2010)) attempts to reply by

\footnotetext{
17 They opt to reject this standard association of symmetry with objectivity (called by them 'invariantism') and propose their so called 'perspectival invariantism', according to which the objectivity is always subject to a choice of an invariance criterion-the choice of which is the given group of transformations - and that choice is a matter of convention.
} 
appealing to the empirical consequences that only the actual chosen structures exhibit: only these structures are those that can be related to physical phenomena. French (2014, 161, his italics) for instance says: "this significance, understood appropriately broadly, is 'explained', again, by the way the world is." However, this is clearly not satisfactory, since obviously some other structures could have been those having empirical significance. His reply begs the question, as we are asking why it is that this is the way the world is structured, so it is unacceptable to answer 'because this is the way the world is structured'.

The problem of the significance of objectivity is thus threatened by the characterization exposed in section 4 , which has led us to a more general threat that the ontic structural realist has to face: the physical significance of some structures over the whole space of mathematical structures. This is just the point that I am going to raise in the next paragraphs without restricting it to OSR, but rather applying it to any metaphysics of fundamental laws.

\subsection{The Fundamental Metaphysics that stems from Symmetry Restoration}

Third and last, the kind of contingency highlighted puts on the table the question of why there are these laws-why these symmetries - and not others. Yet without additional premises, such a contingency does not necessarily add a layer of dissatisfaction to our grasp of the nature of laws, in so far as traditional accounts of lawhood-such as the Necessitarian or the Humean-just plainly accept the contingency of the laws. I argue for this dissatisfaction, though, providing a symmetry argument that appeals to such a characterization. This symmetry argument gives a reason to think that bigger symmetry groups conjectured by ToE's welcome further explanation.

The argument relies not only on the contingency highlighted, but also on the premise that it is asymmetry, not symmetry, that demands explanation. The demand of explanation is formulated in terms of the appearance of having been designed; what seems to have been designed is what asks for further explanation. 
There is a common confusion of associating symmetry with appearance of design and thus with the need of explanation. But is the other way around: Kosso (2003) brushes away the confusion of associating a symmetric universe with the existence of a designer that designed it, arguing that it is just the opposite. The "default structure" is symmetry; "it is the symmetry breaking, not the symmetry, that is the product of design" (Kosso, 2003, 421). This claim goes along the lines of Curie's first principle, which yields that it is asymmetry that creates the phenomena that otherwise would be indifferentiable. We can find Curie's principle stated thus: "When certain effects show a certain asymmetry, this asymmetry must be found in the causes which gave rise to them." (Curie, 1894, 401). Brading and Castellani $(2003,313)$ point out the underlying idea: "Effects are phenomena which always require a certain asymmetry in order to arise. If this asymmetry does not exist, the phenomena are impossible."

Ismael (1997) defends the necessary truth of this principle when properly understood, and shows that instances of spontaneous symmetry breaking such as those that we are here entertaining are not counterexamples to it (see also Castellani and Ismael (forthcoming) and Brading and Castellani (2013, 4.2)). Brading and Castellani $(2013,4.2)$ give us an understanding of Curie's principle when extended to include spontaneous breaking of symmetries: we can maintain that an asymmetry of the phenomena must come from the breaking (explicit or spontaneous) of the symmetry of the fundamental laws.

Crucially, notice now that the "default structure" corresponds to a case of strictly absolute symmetry. Hence, as long as we follow the principle, the unique satisfactory metaphysics of fundamental laws that would not demand explanation would be a situation of absolute dynamical symmetry. This means that the state of the world would have to be invariant with respect to all groups of possible transformations. Rather than being invariant with respect to a specific finite collection of group transformations, such as (say) $S L(2, \mathbb{C}) \otimes S U(3) \otimes S U(2) \otimes U(1)$, the state of the world would have to be invariant with respect to any group of possible transformations. But this will clearly not be the case for any of the ToE's we are entertaining - i.e., those based on QFT and constrained by bigger symmetry 
groups, no matter how big, and no matter which. The state of the world will be invariant with respect to a finite set of symmetry groups, not with respect to all. Hence, bigger symmetry groups will still be asymmetric to some degree, in that they will not correspond to a state of absolute dynamical symmetry. Thus, this leads us to ask for further explanation of the future final laws of a ToE composed of bigger gauge symmetry groups.

In sum, if we follow Kosso (2003), given the relation of asymmetry with design and design in turn with need of explanation, and given the degree of asymmetry still present in future symmetry groups, we conclude that such symmetry groups, qua fundamental laws, welcome further philosophical elucidation.

The underlying rationale of this argument connects with what Weinberg (1981) once illustrated. He envisaged two paths that scientific inquiry could take: explain symmetries or explain their absence. The orthodox attitude in the physics community corresponds to the second of Weinberg's branches, i.e., physics has to explain their absence. ${ }^{18}$ Notice that, having followed Kosso (2003), my argument is to be framed within the orthodoxy; my critical assessment comes from the second of Weinberg's branches-physics has to explain the absence of symmetry.

Additionally, it is interesting to note that the other branch also asks for an explanation of the (same) symmetry groups, although for other reasons. Those who hold that it is symmetry that demands explanation (i.e., those who seek to understand what is permanent in terms of what changes) take as the default natural state something that now would amount to "absolute asymmetry". But what does this mean? This state of absolute asymmetry, which the first of Weinberg's branches takes it to be the only acceptable and natural primitive state, is intended to correspond to the lack of any stable laws of nature. This is in fact the underlying idea in Wheeler's 'law without law', ${ }^{19}$ in C. S. Peirce's motivation of his evolutionary cosmology, ${ }^{20}$ and in several projects in contemporary theoretical

\footnotetext{
${ }^{18}$ This dichotomy is analogous to what Klein and Lachièze-Rey $(1993,11)$ set forth regarding the goal of physics: to study "what changes in terms of what is permanent" or "what is permanent in terms of what changes".

${ }^{19}$ See Wheeler (1982), Wheeler (1983), and a critical assessment in Deutsch (1986).

${ }^{20}$ See Peirce (1867), and a critical assessment in Reynolds (2002).
} 
physics which seek to derive (all) the fundamental symmetry principles from a complex underlying level. ${ }^{21}$ This other branch, then, is also unsatisfied by the metaphysics that stems from current ToE's, but now because they would only be satisfied with (something as) a lawless fundamental level from which symmetries somehow emerge. 22

\section{Conclusion}

I have argued that, despite the remarkable virtues of the new physics portrayed in section 3, the characterization of the laws of such physics laid out in section 4 leads us to consider such laws not in less need of philosophical elucidation than other previous laws of nature. ${ }^{23}$

In section 4 we gave a characterization of gauge symmetries through the analysis of the strong nuclear interaction. We started by pointing out the constraints that determine a free and then an interacting particle. A free particle is only determined by transforming under the space-time symmetry group $\operatorname{SL}(2, \mathbb{C}) \ltimes \mathbb{R}^{3,1}$, and an interacting particle transforms under $S L(2, \mathbb{C}) \ltimes \mathbb{R}^{3,1}$ and a generic infinitedimensional group of gauge transformations - which in our world turns out to be

\footnotetext{
${ }^{21}$ According to the team led by H.B. Nielsen, all complex Lagrangians lead in the low-energy limit to the symmetries of current physics Froggatt and Nielsen (1991, 2002), Chadha and Nielsen (1983), Chkareuli et al. (2011) (see also Mukohyama and Uzan (2013) or Jacobson and Wall (2010) for the case of Lorentz symmetry). They are considering a fundamental level ruled by an undetermined highly complex behaviour, labeled by them as "random dynamics". Also the (speculative) projects of entropic forces should be mentioned, such as that of Verlinde (2011) or the more elaborated derivation of the Einstein field equations from thermodynamic assumptions of Jacobson (1995).

${ }^{22}$ Perhaps the reader has noticed the interesting convergence of the lawless fundamental state of the second branch with the state of absolute dynamical symmetry of the first branch. That is, it could be elsewhere explored that the absolute dynamical symmetry that we would expect following Kosso (2003) amounts to the random dynamics of Froggatt and Nielsen (1991) - in the same sense as the liquid state of water is more symmetric than the frozen snowflake, in spite of the randomness and lack of ordered organization of the molecules in the liquid state.

${ }^{23}$ This conclusion, though, is not intended to discourage the search for ever more encompassing symmetry groups as a principle of heuristics.
} 
$S U(3) \otimes S U(2) \otimes U(1)$. Yet then we saw that a generic interacting particle is strongly underdetermined by these two constraints. Namely, we saw that:

1. The symmetry group $\mathrm{SU}(3)$ associated to the bosons of the strong interaction is chosen due to empirical adequacy from among an infinite space of possible symmetry groups;

2. Unlike the case of bosons (which are uniquely determined by the adjoint representation), the individuation of fermions with an irreducible representation of $\mathrm{SU}(3)$ is itself chosen due to empirical adequacy from among an infinite space of possible irreducible representations;

3. SU(3) even with a fixed representation does not univocally correspond to one possible world - it can at least correspond to a world with eight or with nine gluons;

4. Conversely, there is no a priori reason to prefer one group ( $\mathrm{SU}(3)$ over $\mathrm{U}(3)$ ) in the possible world of nine gluons described above, so that two different groups could be chosen to represent the same state of affairs.

Thus, even if assuming the (disputable) claims laid out in section 2 that any ToE constructed from QFT 1) is asymptotically safe, 2) succeeds in its prospect of unification, 3) exhibits no unnatural parameters, and 4) the process of symmetry breaking is satisfactorily understood, the arguments of section 5 conclude that not only are symmetries neither a priori reasonable nor (logically or metaphysically) necessary, but they are neither unreasonably effective, nor significantly objective, nor absolutely symmetric. And this lack of absolute symmetry, if we follow Curie's $1^{\text {st }}$ principle and Kosso (2003), suggests that bigger local gauge symmetries should not be considered as fully satisfactory primitive dynamical principles of a fundamental metaphysics.

\section{References}

Baker, David John. "Symmetry and the Metaphysics of Physics." Philosophy Compass 5, 12 (2010): 1157-1166.

Bangu, Sorin. "Reifying Mathematics? Prediction and Symmetry Classification." Studies in History and Philosophy of Science Part B 39, 2 (2008): 239-258. 
. "Symmetry." In The Oxford Handbook of Philosophy of Physics, edited by Robert Batterman. OUP USA, 2013, chapter 10.

Borrelli, Arianna. "Naturalness and the hierarchy problem in historical-philosophical perspective.”, 2011. (Talk at DESY Hamburg).

Bottomley, James, and John Baez. "Why are there eight gluons and not nine?", 1996. http: //math.ucr.edu/home/baez/physics/ParticleAndNuclear/gluons.html.

Brading, Katherine, and Elena Castellani. "Symmetry and Symmetry Breaking." In The Stanford Encyclopedia of Philosophy, edited by Edward N. Zalta. 2013. Spring 2013 edition.

Brading, Katherine A., and Elena Castellani. Symmetries in Physics: Philosophical Reflections. Cambridge University Press, 2003.

Bueno, Otávio. "An Inferential Conception of the Application of Mathematics." Noûs 45, 2 (2011): 345-374.

Cao, T. Y. "Gauge Theory and the Geometrization of Fundamental Physics." In Philosophical Foundations of Quantum Field Theory, edited by H. R. Brown, and R. Harré. Oxford University Press, 1988, 117-33.

Castellani, Elena. "Reductionism, Emergence, and Effective Field Theories." Studies in History and Philosophy of Science Part B 33, 2 (2002): 251-267.

- "On the meaning of symmetry breaking." In Symmetries in Physics: Philosophical Reflections, edited by Katherine Brading, and Elena Castellani. Cambridge University Press, 2003, 321-334.

Castellani, Elena, and Jenann Ismael. "Which Curie’s Principle.” Philosophy of Science .

Cat, Jordi. "The physicists' debates on unification in physics at the end of the 20th century." Historical Studies in the Physical and Biological Sciences 253-299.

Chadha, S, and Holger Bech Nielsen. "Lorentz invariance as a low energy phenomenon." Nuclear Physics B 217, 1 (1983): 125-144.

Chkareuli, J.L., C.D. Froggatt, and H.B. Nielsen. "Spontaneously Generated Tensor Field Gravity." Nucl.Phys. B848 (2011): 498-522.

Cottingham, W.N., and D.A. Greenwood. An Introduction to the Standard Model of Particle Physics. Cambridge University Press, 2007.

Curie, Pierre. "Sur la symétrie dans les phénomènes physiques, symétrie d'un champ électrique et d'un champ magnétique." Journal de physique III.

Debs, T.A., and M. Redhead. Objectivity, Invariance, and Convention: Symmetry in Physical Science. Harvard University Press, 2009.

Deutsch, David. "On Wheeler's notion of "law without law" in physics." Foundations of Physics 16, 6 (1986): 565-572.

Earman, John. "Rough guide to spontaneous symmetry breaking." In Symmetries in Physics: Philosophical Reflections, edited by Katherine Brading, and Elena Castellani. Cambridge University Press, 2003, 140-162. 
Evans, Jared, Yevgeny Kats, David Shih, and Matthew Strassler. "Toward full LHC coverage of natural supersymmetry." Journal of High Energy Physics 2014, 7: 101. http://dx.doi. org/10.1007/JHEP07\%282014\%29101.

Feng, Jonathan L. "Naturalness and the Status of Supersymmetry." Ann.Rev.Nucl.Part.Sci. 63 (2013): 351-382.

Filomeno, Aldo. On the Possibility of Stable Regularities Without Fundamental Laws. Ph.D. thesis, Autonomous University of Barcelona, 2014. Isbn:9788449047107.

Fischbach, Ephraim, Daniel Sudarsky, Aaron Szafer, Carrick Talmadge, and S. H. Aronson. "Reanalysis of the Eotvös experiment." Phys. Rev. Lett. 56 (1986): 3-6. http: //link . aps .org/ doi/10.1103/PhysRevLett.56.3.

Fowlie, Andrew. "CMSSM, naturalness and the "fine-tuning price" of the Very Large Hadron Collider.” Phys. Rev. D 90 (2014): 015,010. http://link.aps.org/doi/10.1103/PhysRevD. 90.015010 .

French, Steven. "The Interdependence of Objects, Structure, and Dependence." Synthese 175 (2010): 89-109. 2014.

The Structure of the World: Metaphysics and Representation. Oxford University Press,

French, Steven, and James Ladyman. "Between Platonism and Phenomenalism: Reply to Cao." Synthese 136, 1 (2003): 73-78.

French, Steven, and Kerry McKenzie. "Rethinking Outside the Toolbox: Reflecting Again on the Relationship Between Metaphysics and Philosophy of Physics." In The Metaphysics of Contemporary Physics, edited by Tomasz Bigaj, and Christian Wüthrich. Brill, 2015.

Friederich, Simon. "Gauge symmetry breaking in gauge theories-in search of clarification." European journal for philosophy of science 3, 2 (2013): 157-182.

Friederich, Simon, Robert Harlander, and Koray Karaca. "Philosophical Perspectives on Ad Hoc Hypotheses and the Higgs Mechanism.” Synthese 191, 16 (2014): 3897-3917.

Froggatt, C. D., and H. B. Nielsen. Origin of symmetries. World Scientific, 1991.

_ . "Derivation of Lorentz Invariance and Three Space Dimensions in Generic Field Theory." ArXiv High Energy Physics - Phenomenology e-prints arXiv : hep-ph/0211106.

Griffiths, D. Introduction to Elementary Particles. Physics textbook. Wiley, 2008. http:// books . google. com/books?id=w9Dz56myXm8C.

Hellman, Geoffrey. Mathematics without numbers: towards a modal-structural interpretation. Clarendon Press, 1989.

Ismael, Jenann. “Curie’s Principle.” Synthese 110, 2 (1997): 167-190.

Ismael, Jenann, and Bas C. Van Fraassen. "Symmetry as a guide to superfluous theoretical structure." In Symmetries in Physics: Philosophical Reflections, edited by Katherine Brading, and Elena Castellani. Cambridge University Press, 2003, 371-392.

Jacobson, Ted. "Thermodynamics of space-time: The Einstein equation of state." Phys.Rev.Lett. 75 (1995): 1260-1263. 
Jacobson, Ted, and A. C. Wall. "Black Hole Thermodynamics and Lorentz Symmetry." Found.Phys. 40 (2010): 1076-1080.

Kantorovich, Aharon. "The Priority of Internal Symmetries in Particle Physics." Studies in History and Philosophy of Science Part B 34, 4 (2003): 651-675.

. "Ontic Structuralism and the Symmetries of Particle Physics." Journal for General Philosophy of Science / Zeitschrift für Allgemeine Wissenschaftstheorie 40, 1 (2009): 73-84.

Klein, Etienne, and Marc Lachièze-Rey. The Quest for Unity. Oxford University Press, 1993.

Kosso, P. "The Empirical Status of Symmetries in Physics." British Journal for the Philosophy of Science 51, 1 (2000): 81-98.

Kosso, Peter. "Symmetry, objectivity, and design.” In Symmetries in Physics, edited by Katherine Brading, and Elena Castellani. Cambridge University Press, 2003, 413-424. Cambridge Books Online.

Ladyman, James. "Structural Realism." In The Stanford Encyclopedia of Philosophy, edited by Edward N. Zalta. 2014. Spring 2014 edition.

Ladyman, James, and Don Ross. Every Thing Must Go: Metaphysics Naturalized. Oxford University Press, 2009.

Lederman, L.M., and C.T. Hill. Symmetry and the beautiful universe. Prometheus Books, 2004.

Lyre, Holger. "Holism and Structuralism in U(1) Gauge Theory." Studies in History and Philosophy of Science Part B 35, 4 (2004): 643-670.

Martin, Christopher. "On Continuous Symmetries and the Foundations of Modern Physics." In Symmetries in Physics: Philosophical Reflections. Cambridge University Press, 2003, 29-60.

Maudlin, Tim. "On the Unification of Physics." Journal of Philosophy 93, 3 (1996): 129-144.

McCabe, Gordon. The structure and interpretation of the standard model. volume 2 of Philosophy and Foundations of Physics. Elsevier, 2011.

McKenzie, Kerry. "On the Fundamentality of Symmetries." In Philosophy of Science PSA proceedings. 2012.

. "In No Categorical Terms: A Sketch for an Alternative Route to Humeanism about Fundamental Laws." In New Directions in the Philosophy of Science, edited by M. Weber W. Gonzalez D. Dieks M. C. Galavotti, S. Hartmann, and T. Uebel. Springer, 2013.

_. "Priority and Particle Physics: Ontic Structural Realism as a Fundamentality Thesis." British Journal for the Philosophy of Science 65, 2 (2014): 353-380.

Morrison, Margaret. "The New Aspect: Symmetries as Meta-Laws.” In Laws of Nature: Essays on the Philosophical, Scientific and Historical Dimensions, edited by Friedel Weinert. Walter De Gruyter, 1995, 157-190.

\footnotetext{
bridge Univ Pr, 2000.
} 
_. "Spontaneous symmetry breaking: Theoretical arguments and philosophical problems." In Symmetries in Physics: Philosophical Reflections, edited by Katherine Brading, and Elena Castellani. Cambridge University Press, 2003, 347-363.

. "Unification in Physics." In The Oxford Handbook of Philosophy of Physics, edited by R. Batterman. OUP USA, 2013, chapter 11.

Mukohyama, Shinji, and Jean-Philippe Uzan. "From configuration to dynamics: Emergence of Lorentz signature in classical field theory." Phys.Rev. D87, 6 (2013): 065,020.

Nounou, Antigone M. "For or Against Structural Realism? A Verdict From High Energy Physics." Studies in History and Philosophy of Science Part B: Studies in History and Philosophy of Modern Physics 49 (2015): 84-101.

Nozick, Robert. Invariances: The Structure of the Objective World. Harvard University Press, 2001.

Peirce, C. S. The Essential Peirce, Volume 1: Selected Philosophical Writings (1867-1893). Indiana University Press, 1867, 1992 edition.

Pincock, Christopher. "A Revealing Flaw in Colyvan's Indispensability Argument." Philosophy of Science 71, 1 (2004): 61-79.

Räz, Tim, and Tilman Sauer. "Outline of a Dynamical Inferential Conception of the Application of Mathematics." Studies in History and Philosophy of Science Part B: Studies in History and Philosophy of Modern Physics 49 (2015): 57-72.

Redhead, M. L. G. "The interpretation of gauge symmetry.” In Symmetries in Physics: Philosophical Reflections. Cambridge University Press, 2003, 124-140.

Reynolds, A. Peirce's Scientific Metaphysics: The Philosophy of Chance, Law, and Evolution. The Vanderbilt Library of American Philosophy Series. Vanderbilt University Press, 2002.

Rickles, Dean, and Steven French. "Understanding permutation symmetry." In Symmetries in Physics: Philosophical Reflections, edited by Katherine Brading, and Elena Castellani. Cambridge University Press, 2003, 212-238.

Roberts, J.T. The Law-Governed Universe. Oxford University Press, 2008.

Robinson, Matthew B., Karen R. Bland, Gerald B. Cleaver, and Jay R. Dittmann. "A Simple Introduction to Particle Physics. Part I - Foundations and the Standard Model.” .

Ross, Don, James Ladyman, and Harold Kincaid. Scientific Metaphysics. Oxford University Press $\mathrm{Uk}, 2015$.

Shapiro, Stewart. Philosophy of Mathematics: Structure and Ontology. Oxford University Press, 1997.

Simon, Barry. Representations of Finite and Compact Groups. Graduate studies in mathematics. American Mathematical Society, 1995.

Strocchi, Franco. "Spontaneous symmetry breaking in quantum systems." Scholarpedia 7, 1 (2012): 11,196.

Tahko, Tuomas E., and Matteo Morganti. "Moderately Naturalistic Metaphysics.” Synthese 1-24. 
Tegmark, M. Our Mathematical Universe: My Quest for the Ultimate Nature of Reality. Alfred A. Knopf. Random House Incorporated, 2014.

Unger, R.M., and L. Smolin. The Singular Universe and the Reality of Time. Cambridge University Press, 2014

Van Fraassen, Bastian. Laws and Symmetry. Oxford University Press, 1989.

Verlinde, Erik P. “On the Origin of Gravity and the Laws of Newton.” JHEP 1104 (2011): 029.

Weinberg, Steven. "Conceptual foundations of the unified theory of weak and electromagnetic interactions." Selected Papers on Gauge Theory of Weak and Electromagnetic Interactions 1.

Weyl, Hermann. Symmetry. Princeton University Press, 1952.

Wheeler, John Archibald. "Law without law." In Quantum Theory and Measurement, edited by Wojciech Hubert Zurek, and John Archibald Wheeler. Princeton University Press, 1982, 182213.

. "On recognizing 'law without law', Oersted Medal Response at the joint APS-AAPT Meeting, New York, 25 January 1983.” American Journal of Physics 51 (1983): 398.

Wigner, Eugene. "The Unreasonable Effectiveness of Mathematics in the Natural Sciences." Communications on Pure and Applied Mathematics 1, 13 (1960): 114.

—. Symmetries and Reflections. Bloomington, IN: Indiana University Press, 1967.

Wilczek, F. The Lightness of Being: Mass, Ether, and the Unification of Forces. Basic Books, 2008.

Williams, Porter. "Naturalness, the Autonomy of Scales, and the $125 \mathrm{GeV}$ Higgs." Studies in History and Philosophy of Science Part B: Studies in History and Philosophy of Modern Physics 51 (2015): 82-96. 